\title{
Giovanni Busino
}

professeur honoraire à l'Université de Lausanne

(23 octobre 2004)

\section{"Lire Pareto aujourd'hui"}

Un document produit en version numérique par Jean-Marie Tremblay, bénévole, professeur de sociologie au Cégep de Chicoutimi

Courriel: jean-marie tremblay@uqac.ca

Site web pédagogique $:$ http://www.uqac.ca/jmt-sociologue/

Dans le cadre de la collection: "Les classiques des sciences sociales"

Site web: http://www.uqac.ca/Classiques_des_sciences_sociales/

Une collection développée en collaboration avec la Bibliothèque Paul-Émile-Boulet de l'Université du Québec à Chicoutimi

Site web: http://bibliotheque.uqac.ca/ 
Cette édition électronique a été réalisée par Jean-Marie Tremblay, bénévole, professeur de sociologie au Cégep de Chicoutimi à partir de l'article de :

\section{Giovanni Busino professeur honoraire à l'Université de Lausanne}

\section{"Lire Pareto aujourd'hui"}

Un article publié pour Les Classiques des sciences sociales, dans Liens-Socio, à l'occasion de la diffusion de la version numérique du Traité de sociologie générale de Vilfredo Pareto, le 23 octobre 2004.

[Autorisation accordée, le 9 août 2005, par M. Jean-Claude Passeron, sociologue à l'EHESS, de diffuser ce texte écrit pour Les Classiques des sciences sociales en 2004. M. Passeron nous confirme l'autorisation de M. Busino.]

Polices de caractères utilisée :

Pour le texte: Times New Roman, 14 points.

Édition électronique réalisée avec le traitement de textes Microsoft Word 2004 pour Macintosh.

Mise en page sur papier format LETTRE (US letter), 8.5' x 11',)

Édition complétée le 14 août 2005 à Chicoutimi, Ville de Saguenay, province de Québec. 


\section{Giovanni Busino}

professeur honoraire à l'Université de Lausanne

\section{"Lire Pareto aujourd'hui" (2004)}

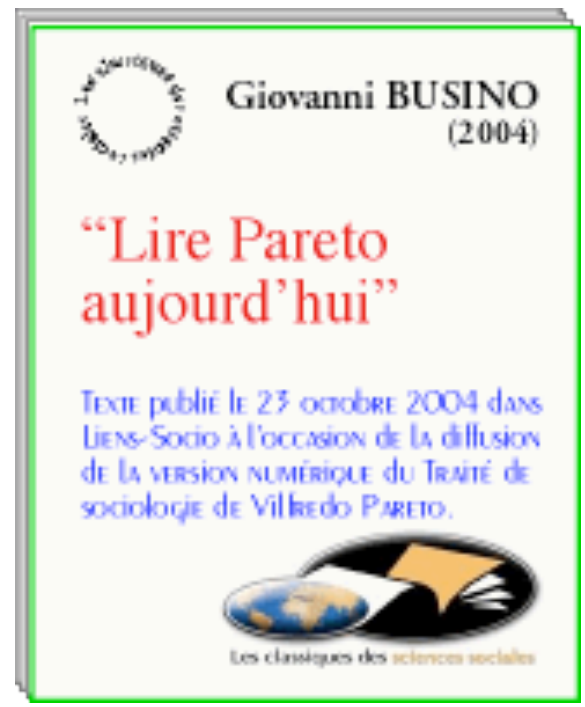

Un article publié pour Les Classiques des sciences sociales, dans Liens-Socio, à l'occasion de la diffusion de la version numérique du Traité de sociologie générale de Vilfredo Pareto, le 23 octobre 2004. 


\title{
"Lire Pareto aujourd'hui"
}

\author{
par Giovanni Busino
}

23 octobre 2004

Giovanni Busino est professeur honoraire à l'Université de Lausanne. Il est l'éditeur des Euvres complètes de Vilfredo Pareto aux Editions Droz. Il est notamment l'auteur de: Sociologies des sciences et des techniques (Paris, Presses Universitaires de France, Que sais-je ?, 1998) ; Paroles reçues : du bon usage des sciences sociales (avec Gérald Berthoud, Genève, Droz, 2000); La sociologie durkheimienne, tradition et actualité : à Philippe Besnard, in memoriam (textes et études réunis par Massimo Borlandi et Giovanni Busino, Genève, Paris, Droz, 2004). [Sources : Liens-Socio]

Parmi les classiques de la sociologie la place de Vilfredo Pareto est toujours contestée et a soulevé depuis un siècle nombre de réserves importantes. En France par exemple, Raymond Aron a écrit que Pareto ne jouirait jamais, sur cette terre, d'une reconnaissance unanime et pacifique; Raymond Boudon est convaincu que le purgatoire de la sociologie parétienne risque de se prolonger encore un peu; et il ne manque pas d'auteurs qui, comme Henri Mendras, considèrent que le savant italo-suisse ne peut pas être rangé parmi nos pères fondateurs car son oeuvre n'apporte guère d'aide intellectuelle ou d'instrument méthodologique aux chercheurs contemporains. On peut aussi s'étonner, avec Jean-Claude Passeron, que le tarissement des passions idéologiques du $\mathrm{XIX}^{\circ}$ siècle n'ait pas ramené plus vite l'attention des sociologues sur la théorie sociologique la plus originale, parmi celles qui s'attachent à unifier l'ensemble des sciences sociales. 
En vérité, les raisons de cet état de fait sont singulières. Les critères employés pour qualifier un sociologue de « classique » pourraient, en effet, s'il n'y avait d'autres anguilles sous roche, s'appliquer sans problème à Pareto. Ses analyses de la société européenne de 1870 à 1923 sont utilisées avec profit par les historiens de différents pays ; les innombrables lectures de ses travaux ont donné naissance à une montagne d'exégèse - dont la conflictualité même souligne sa place privilégiée au cœur des débats scientifiques et idéologiques du début du $\mathrm{XX}^{\circ}$ siècle ; elles sont aussi au principe de re-formulations théoriques importantes chez des auteurs notables, en particulier dans le domaine de la sociologie politique ou à propos du lien qu'il établissait entre sociologie et économie ; enfin ses concepts et ses schèmes d'analyse sont aujourd'hui utilisés par des chercheurs de terrain, parfois éloignés de ses options épistémologiques de base : Middletown III Research par exemple, à laquelle Theodore Caplow a activement participé comme les innombrables enquêtes sur les élites dont John Scott a tracé l'historique dans trois gros volumes parus en 1990, en fournissent quelques aperçus probants. Pourquoi alors cet étrange, pour ne pas dire bizarre destinée ? Le caractère abrupt de Pareto, son goût de la polémique, parfois de la provocation, ne suffisent pas à expliquer sa marginalité dans la cité savante, cela d'autant moins que cette réserve de la postérité concerne presque exclusivement Pareto sociologue, l'économiste ayant au contraire pris rang par le Manuel d'économie politique, comme par le Cours d'économie politique parmi les fondateurs de la théorie néo-classique de l'équilibre.

Les réponses données jusqu'ici à la question posée par la réception cacophonique de l'œuvre de Pareto me paraissent anecdotiques et parfois franchement contradictoires. Pareto n'est pas le seul sociologue outsider à débattre - en affichant une neutralité éthique, où perce sans doute une certaine sympathie pour la recherche "réaliste » de l'efficacité politique ; mais moins que chez Machiavel à qui nul ne dispute la lucidité scientifique dont ce «cynisme » politique est le prix - de la force et de la violence, de la ruse ou du mensonge ; ni non plus le seul à inscrire ses thèses dans des livres monstrueux, hermétiques, mélangeant les genres scientifiques, nourris de surcroît de ses lectures minutieuses de toutes les rubriques de presse ; comme il n'était pas le seul, en cette fin du $\mathrm{XIX}^{\circ}$ siècle à narguer le conformisme des bienpensants, ou même à braver les règles et les conventions de la communauté scientifique à laquelle il revendiquait d'appartenir, mais en voltigeant d'une discipline à l'autre et en gardant un attachement littéraire à la culture classique de l'Antiquité. Au tournant de l'avantdernier siècle, il n'a pas, non plus, été le seul savant à laisser percer quelques faiblesses pour les régimes politiques autoritaires, bref à af- 
ficher son cynisme d'analyste politique et son pessimisme de philosophe de la nature humaine.

En revanche, il est assurément le seul, dans l'histoire de la discipline, à rejeter, à la fois et sans ménagement, le patrimoine commun des valeurs chrétiennes, le positivisme qui débouchait sur une morale humaniste du progrès, comme l'application de l'utilitarisme à l'explication de toutes les action sociales. Il est encore le seul à récuser le rêve philosophique d'une "objectivité » intrinsèque de la science, ou le mythe d'une "rationalité » inhérente au cours du monde historico-social, en somme, à se camper ostentatoirement à l'opposé des options théoriques fondatrices de la sociologie. Il joint à une conceptualisation ésotérique formulée dans un langage volontairement sibyllin (ainsi pour le terme d' "ophélimité » préféré à celui trop équivoque, selon lui, d' " utilité ») des choix inattendus d'objets ou d'hypothèses (sur la décadence d'un pouvoir ou la circulation des élites par exemple), qui le démarquent avec éclat de la lettre et de l'esprit des paradigmes déjà classiques, tel que celui de l'efficacité universelle du principe de rationalité, à laquelle il oppose la force des sentiments collectifs, l'irrationalité des conflits historiques les plus lourds de conséquences durables et les effets persuasifs de l'usage délirant ou enfantin de la logique (5ème classe des résidus).

L'écart entre les conceptualisations sociologiques de Pareto et celles des principales tendances de la théorie sociale au $\mathrm{XX}^{\circ}$ siècle était, sans nul doute, considérable. Mais sa doctrine accueillait aussi des hybridations complexes. On a dit Pareto proche de l'école politologique italienne qui formulait, avec Mosca, la célèbre «Loi d'airain des oligarchies ». Certes. Mais les écrits et la correspondance montrent aussi le militant «libéral » attaché à toutes les formes de la liberté d'expression, soucieux de transparence dans la vie publique, en quête d'une organisation économico-sociale décentralisée, loin des dogmes politiques, socialistes ou capitalistes : ainsi, entre la nationalisation et la privatisation d'entreprises à vocation nationale, telles les compagnies des chemins de fer, Pareto considérait que la solution la plus efficace serait leur transformation en coopératives confiées aux soins des cheminots, c'est-à-dire aux travailleurs eux-mêmes. L'extrême complexité et la mobilité incessante des actions humaines poussait le libéral Pareto, souvent insolent avec les doctrines sociales qui lui semblaient démagogiques, à prendre lui-même une position inattendue vis-à-vis de ses propres opinions politiques, parfois aussi critique que vis-à-vis de celles de ses adversaires, en particulier à propos de la démocratie. Ainsi pouvait-il considérer la démocratie comme un bien social - autrement doit comme le moindre mal politique possible - et pour cela inviter continuellement les citoyens à la raison et à la 
« prudence », tout en rappelant qu'il n'y a pas de société sans une capacité virtuelle à user de la force, c'est-à-dire sans la détermination de l'utiliser lorsqu'il est indispensable de le faire : ceux qui professaient un pacifisme inconditionnel, pour des raisons religieuses ou morales, lui paraissent prêts à devenir des esclaves.

Exemple plus central de sa position atypique. Alors même que le socle épistémologique de sa sociologie était positiviste par sa conception du raisonnement scientifique comme rapport entre une pure et simple observation des répétitions de faits et une formulation par induction des généralités qui les subsument - ce qui revient à une affirmation, presque scientiste, de l'inutilité de toute théorie préalable dans une science logico-expérimentale, épistémologie qu'il détaillait longuement dans les " Préliminaires » méthodologique au Traité de sociologie générale - Pareto parle du " positivisme » historique, celui des penseurs et sociologues de son époque qui en tiraient une morale optimiste du progrès, comme d'une croyance métaphysique, d'un dogmatisme indifférent à l'observation pratiquée sans idée préconçue, d'un mélange artificiel d'intérêts cognitifs et d'utilités sociales, d'être et de devoir-être, de théorie et de pratique, de science et de réformisme social. Comte, Spencer, Durkheim, et bien d'autres, sont qualifiés de fidèles de la "religion humanitaire »; leur prétention à pratiquer une science sociale ouvrant sur des réformes taxée d'incongruité intellectuellement fallacieuse et socialement dangereuse.

Dans ces conditions, les difficultés que j'ai rencontrées pour entreprendre, il y a quelques quarante ans, et, bon an mal an mener à terme le rassemblement et l'édition de l'essentiel de la production scientifique et littéraire de Pareto n'ont rien pour étonner dans un monde intellectuel qui, à l'échelle internationale, était traversé à propos de Pareto par des dénonciations et des affiliations également violentes ou sectaires. Entré tardivement dans le microcosme parétien, je fus immédiatement impressionné par ce qui s'y passait. Il existait alors, au plan international, une véritable secte parétienne, avec ses rites et ses liturgies, avec ses cardinaux gardiens sourcilleux de l'orthodoxie, avec son service d'assistance, et même sa banque (le "Pareto Fund » de New York), très peu préoccupée par ce qu'il se passait ailleurs dans le monde. J'ai alors pu observer les activités des parétologues, tout en restant un agnostique, ni adepte ni compagnon de voyage. Etudier Pareto était pour moi un moyen d'étudier l'histoire des sciences sociales, en Suisse et en Europe, de redécouvrir des filons de la vie intellectuelle et éthico-politique négligés dans la péninsule italienne et dans les pays francophones. Mais la plus grande part de la littérature consacrée à Pareto me laissait perplexe. Les écrits des parétologues étaient presque toujours hagiographiques et a-critiques, insensibles au renou- 
vellement des implications sociales de la sociologie et de l'économie comme aux problématiques scientifiques contemporaines. Ceux des non-affiliés, qui n'avaient pas lu, ou mal lu Pareto, se prévalaient d'une ou de quelques thèses parétiennes dé-contextualisées pour consolider une autre foi, défendre une autre perspective idéologique, et toujours pour vanter ou pour discréditer, dans un esprit partisan, une théorie ou une doctrine. Dans les deux cas, il me semblait que la critique des textes, tant interne qu'externe, n'était guère respectée.

Ce qui me frappa d'abord, en effet, c'est que la lecture des textes garantie par des éditions correctes, conditions généralement assurées pour la plupart des pères fondateurs des sciences sociales, se révélait presque impossible pour Pareto. Une grande partie des textes de cet auteur était introuvable, et ceux qui se trouvaient disponibles étaient souvent expurgés ou adultérés. La première opération nécessaire ressortait de l'historiographie des textes et d'une critique philologique. Je crois avoir à l'époque, mais peut-être avec trop d'irrespect à l'égard des travaux accomplis par mes prédécesseurs, exprimé toutes mes réserves dans un travail qui présentait une revue critique des travaux consacrés à Pareto, et plus particulièrement des études parues entre 1960 et 1965, et qui figure aujourd'hui dans le volume Gli studi su Vilfredo Pareto oggi : Dall'agiografia alla critica, 1923-1973 (Roma, Bulzoni, 1974). D'où déclenchement d'une vive polémique où les parétiens m'accusèrent de déloyauté, de "sauvagerie » même, tandis que d'autres, en Italie - mais aussi ailleurs puisque les échos de ce débat s'étendirent jusqu'aux colonnes d'un quotidien nippon à grand tirage - accusèrent l'entreprise de réédition de monopoliser en faveur d'une institution helvétique les droits d'auteur sur les œuvres parétiennes.

Les autres difficultés furent évidemment financières, institutionnelles et universitaires étant donné les exigences d'une récollection aussi vaste, bien qu'il ne se soit agi, en somme, que d'une édition que j'appelais «préparatoire ». Elles expliquent que l'on n'ait pu respecter l'ordre chronologique ou que certains articles aient été publiés plusieurs fois, en des versions différentes. En tout cas le programme que nous nous étions fixé est désormais en voie d'achèvement. En gros, tous les écrits de Pareto rédigés en français, en anglais, en allemand et en espagnol se retrouvent dans les volumes édités par Droz, où seules les études rédigées directement en italien figurent en cette langue dans notre édition. Mais d'ores et déjà on peut affirmer que quatre-vingts ans après sa mort, "tout Pareto » a été réuni et republié : les œuvres principales, les écrits mineurs, une partie importante de sa correspondance scientifique et personnelle. Il faut ajouter qu'à la fin de 1996 Christie's a mis en vente les 20 volumes (environ 10.000 pages ma- 
nuscrites) dans lesquels Pareto transcrivait toutes ses lettres, de 1874 à 1923 (à une interruption près) : comme nous nous avons là, pour la première fois dans l'histoire des sciences sociales, la totalité de la correspondance d'un sociologue, il est certain que cette documentation offrira, lorsque elle sera édité, une image enfin complète de la vie et des œuvres de Vilfredo Pareto. Tout cela nous permet de formuler aujourd'hui un jugement plus serein sur cet auteur « maudit» de la tradition sociologique.

En France, ces dernières décennies, les études ou les relectures de Raymond Aron, de Julien Freund, de Raymond Boudon, de Bernard Valade, d'Alban Bouvier, de Jean-Claude Passeron ou de Marc Barbut sont là pour nous prouver que quelque chose a changé dans la réception de la sociologie de Pareto, depuis les temps de Célestin Bouglé, Maurice Halbwachs et Georges Gurvitch, où le mépris et l'indifférence étaient seuls de mise. Nul doute que les « Oeuvres complètes " n'aient offert un instrument de travail à ceux qui voulaient prendre la mesure de la pensée de Pareto en la situant « dans le cadre du développement de la sociologie et de sa constitution en discipline rigoureuse » ainsi que le résumait Julien Freund. Elles ont peut-être aussi joué un rôle dans le revival parétien qui semble prendre quelque ampleur et dont la bibliographie internationale des sciences sociales marque la diffusion .

Ce retour de Pareto dans l'actualité est sans doute fort différent du revival wébérien observable lui aussi en France dans la même période. Il consiste moins dans une impossible reprise du programme parétien, que dans une remise en discussion de tous les aspects théoriques, historiques et méthodologiques de sa pensée. Pareto a largement profité du retour actuel aux « classiques » et à la tradition sociologique après les excès d'un empirisme sans perspectives et d'un néo-positivisme sans travaux de terrain. D'auteur lu et discuté il y a quarante ans par des chercheurs qui même grands restaient isolés - et comment ne pas rappeler ici la mémoire de Georges-Henri Bousquet, de Jules Monnerot, de Raymond Aron, de Julien Freund, ou ne pas saluer la vitalité gaillarde de Maurice Allais ? - Pareto est devenu, dès les années 80, et d'abord grâce à l'économiste Edmond Malinvaud un social scientist lu, discuté, critiqué mais ré-utilisé comme tel sur une scène désormais mondiale. Une nouvelle image de la sociologie de Pareto s'est ainsi affirmée de plus en plus clairement. Aux panégyriques et aux charges outrancières ont succédé les études minutieuses, érudites, certes attentives aux déficiences de forme ou de fond, mais soucieuses surtout de dégager les traits saillants d'une construction théorique toujours porteuse d'idées, d'hypothèses, de procédés utilisables par toutes les sciences sociales. Quant aux pistes d'analyse politique qui se dégagent 
du Traité on voit mieux, avec le recul historique que procure depuis peu l'histoire du marché mondial comme la reconfiguration des crises internationales - sous une forme imprévue après le gel des alliances durant le long conflit bipolarisé entre l'Est et l'Ouest - que le bouleversement des équilibres géopolitiques et des stratégies guerrières, tend à redonner tout son sens à l'analyse parétienne des sentiments et de leurs dérivations, des intérêts et de leurs masques qui sont mis en jeu dans les luttes entre groupes et nations, au détriment des analyses évolutionnistes et linéaires qu'avait favorisés chez ses sociologues ou historiens le demi siècle écoulé de guerres idéologiques.

Il suffit de consulter quelques grands Congrès ou Colloques de ces dernières années, pour voir surgir la multiplicité des questions que posent aujourd'hui toutes les sciences sociales au corpus parétien comme la pertinence de celles que l'on peut poser à nos sociétés à partir des concepts et des analyses qu'il nous a proposées; de même qu'on peut y constater le caractère de plus en plus international des participations de spécialistes. Le congrès, organisé par l'Université de Pise en mai 1996 - Actes dans la revue History of Economic Ideas, 1997 - essayait, par exemple, de tracer une séparation radicale entre Pareto économiste et Pareto sociologue : le premier auteur d'un paradigme rationaliste, le second s'enfermant dans les paradoxes et les ambiguïtés. Quatre ans auparavant, en novembre 1992, l'Université de Trente avait consacré un colloque à l'étude des choix rationnels et des sentiments dans les œuvres du sociologue de Lausanne. Publiés en 1994, les travaux de ce colloque prenaient au contraire la théorie sociologique des émotions, de leur dynamique et de leur forme construite par Pareto, comme exemple de la. « mutuelle dépendance » des rapports entre la raison et les émotions dans les déterminants d'une action sociale ; s'y trouvait aussi posée, de manière critique, la question de la différence de traitement que Pareto réservait aux analogies mécanistes et organicistes en privilégiant les premières.

Le Colloque d'octobre 1997 à la Sorbonne a illustré la longue liste des études sociologiques, historiques méthodologiques ou linguistiques qui pouvaient être entreprises, grâce à l'originalité des thèses parétiennes, sur la rationalité et les logiques naturelles ou scientifiques, sur les rapports entre nature et culture, sur la formation et la transmission des idées, des croyances et sur les procédures rhétoriques ou formelles de persuasion qui les rendent possibles, plausibles, recevables et acceptables. Comme on le voit dans ce colloque, les études de la dernière décennie sur Pareto portent d'abord la marque des travaux de Raymond Boudon du fait même qu'ils ont été diffusés et interprétés différemment ici et là. Les études en langue française s'inspirent ou radicalisent une des trois interprétations boudoniennes : celle des an- 
nées avant 1986, rationaliste et intellectualiste ; une deuxième qui met en avant le recours aux motivations irrationnelles et émotives; puis une troisième qui entend distinguer chez Pareto une sociologie ésotérique d'une autre, exotérique : thèse reprise en Italie par exemple par Giovanni Barbieri, qui l'a portée à ses conséquences extrêmes. Leur succession, qui correspond à l'évolution des thèses épistémologiques de Boudon lui-même, nous propose à travers un déplacement complexe des niveaux d'analyse, parfois un rien sophistiqué, une interprétation de la sociologie de Pareto, qui reste foncièrement rationaliste et intellectualiste. Un article récent d'Alban Bouvier sur le paradigme Mill-Pareto me conforte dans cette conviction.

À la fin novembre de la même année 1997, d'autres chercheurs français, suisses et italiens confrontaient aussi leurs travaux, sous les auspices de 1'Université de Turin et de la Fondation Luigi Einaudi publiés en 2000 sous le titre Economia, sociologia e politica nell'opera di Vilfredo Pareto (Olschki, Firenze). Là aussi la diversité des angles d'attaque révélait la multiplicité des facettes scientifiques qu'offre à la réflexion actuelle l'ensemble des chantiers ouverts par Pareto en sciences sociales. À défaut de les passer en revue, retenons au moins des éclaircissements inattendus sur les influences subies et exercées ; l'examen de théories parétiennes laissées inachevées, par exemple la théorie monétaire, celle de la corruption ou celle du rôle de l'Etat dans la vie économique. Le congrès de Turin a, en outre, fait une large part à la sociologie politique de Pareto et à sa philosophie libertaire, sujets davantage travaillés dans les départements de science politique des Etats-Unis et d'Angleterre qu'en Europe. Cependant la majeure partie des contributions ont porté sur des thèmes jusqu'ici surtout explorés en France comme le rôle des métaphores et des analogies dans le raisonnement sociologique, ainsi que ceux de la méthode historique, des mathématiques et de la formalisation dans les différentes sociales qu'a pratiquées Pareto. D'où a ressurgi au centre du débat la vetustissima ac vexativa quaestio du rapport entre choix rationnels et normes sociales, c'est-à-dire la question des rapports entre économie et sociologie, entre elles et avec l'histoire, où je me contenterai de citer - peut-être parce que je me suis toujours senti (au moins) aussi proche de Max Weber que de Pareto le rapprochement qu'a présenté dans ce colloque Jean- Claude Passeron, à partir de certaines de ses thèses antérieures : "Pareto et Weber, écrit-il, ont un commun d'avoir refusé à la fois le rien et le tout. Ils n'ont voulu ni congédier le principe de rationalité comme principe organisateur de l'enquête sociologique sur les configurations et les déroulements historiques, ni transformer cet opérateur méthodologiquement privilégié $\mathrm{du}$ questionnement des actions sociales en principe d'enfermement théorique $\gg$. 
On est loin aujourd'hui du temps des polémiques entre gardiens ombrageux de l'orthodoxie parétienne et sociologues, humanistes ou marxistes, qui voulaient bannir Pareto de la cité scientifique : si ces derniers affrontaient à tout bout de champ leurs concepts irréconciliables, il interrompaient volontiers le combat pour vouer le sociologue de Céligny à l'Enfer des bibliothèques sociologiques. Si l'on peut emprunter aux Souvenirs d'Alexis de Tocqueville sa définition des grandes théories pour parler d'un tournant de l'histoire des théories à la fin du $\mathrm{XX}^{\circ}$ siècle, disons avec lui que, à partir des années 80, « Les "systèmes absolus" qui font dépendre tous les événements de l'histoire de grandes causes premières se liant les unes aux autres par une chaîne fatale, et qui suppriment, pour ainsi dire, les hommes de l'histoire du genre humain », ces « sublimes théories » ces grands paradigmes qui avaient nom marxisme, structuralisme, fonctionnalisme et quelques autres systèmes en -ismes, ont cédé la place à des cadres de recherche pluralisés, révisables et différemment mobilisables selon les enquêtes, particularisés selon les terrains et les types d'action sociale, dans lesquels on comprend mieux les faisceaux de déterminations interdépendantes qui orientent les visées et les rationalisations des acteurs historiques en fonction des contextes locaux de leurs actes.

Devenue possible aujourd'hui, une lecture sereine, distante, critique des écrits de Pareto nous montre qu'il est bien un classique de la sociologie, l'un de ses pères fondateurs. Le "rationalisme de méthode » propre à la sociologie (comme le nommait Weber pour le distinguer du rationalisme de doctrine) - la méthode que Pareto appelait « logico-expérimentale »- est plus que jamais inscrite, en dépit de la diversité croissante des théories sociologiques, au cœur de la recherche contemporaine.

Giovanni Busino 